\title{
Review Article \\ Concomitant Thyroid Disorders and Inflammatory Bowel Disease: A Literature Review
}

\author{
Toru Shizuma \\ Department of Physiology, School of Medicine, Tokai University, 143 Shimokasuya, Isehara, Kanagawa 259-1193, Japan \\ Correspondence should be addressed to Toru Shizuma; shizuma@is.icc.u-tokai.ac.jp
}

Received 27 November 2015; Revised 30 January 2016; Accepted 16 February 2016

Academic Editor: Luca Elli

Copyright (C) 2016 Toru Shizuma. This is an open access article distributed under the Creative Commons Attribution License, which permits unrestricted use, distribution, and reproduction in any medium, provided the original work is properly cited.

\begin{abstract}
The aim of this report was to review and summarize the literature on cases of concomitant inflammatory bowel disease (IBD) and thyroid diseases. We included the following previous case reports of concomitant IBD and thyroid diseases: 16 cases of ulcerative colitis (UC) and Graves' disease (GD), 3 cases of Crohn's disease (CD) and GD, 10 cases of CD and Hashimoto's thyroiditis (HT), 4 cases of IBD and subacute thyroiditis (SAT) or SAT-like symptoms, and 13 cases of IBD (12/13 cases were CD) and amyloid goiter. There might be no obvious differences of prevalence of thyroid dysfunction (hyper- or hypothyroidism), GD, and thyroid cancer between IBD patients and general populations. However, concomitant UC and HT might be relatively common in patients with multiple autoimmune disorders, and AG is one of the complications with CD patients. There might be no obvious differences of fatal prognoses between IBD patients with thyroid diseases and patients with thyroid diseases without IBD.
\end{abstract}

\section{Introduction}

Ulcerative colitis (UC) and Crohn's disease (CD) are the two most common inflammatory bowel diseases (IBD). Both UC and $\mathrm{CD}$ are chronic recurrent conditions and are characterized by intestinal inflammation that may result from a combination of causes such as environmental or immunological factors [1]. CD can affect any part of the gastrointestinal tract, whereas UC is characterized by inflammation confined to the large intestine [1]. Microscopically, CD affects the entire bowel wall, whereas UC is restricted to the epithelial lining of the gut [1]. Although the development of extraintestinal manifestations or coexistence of autoimmune disorders during the course of IBD is well known, the coexistence of IBD and thyroid diseases has not been well documented.

Thyroid diseases include autoimmune thyroid diseases (ATDs), malignancy, amyloid goiter (AG), subacute thyroiditis (SAT), and congenital thyroid diseases. Thyroid dysfunctions are generally classified into hyperthyroidism or thyrotoxicosis and hypothyroidism. The most common causes of hyperthyroidism are Graves' disease (GD or Basedow disease), excessive supplementation of thyroid hormones, toxic adenoma, and toxic multinodular goiter; nonthyroid diseases may also cause hyperthyroidism [2,3]. On the other hand, the common causes of hypothyroidism are Hashimoto's thyroiditis (HT or autoimmune thyroiditis), iodine-deficient conditions, postoperative states such as thyroidectomy, and isotope treatment; however, thyroid hormone levels in HT patients often display normal levels [4].

To date, there have been few systematic literature reviews of cases of concomitant thyroid diseases and IBD. Here we conducted a literature search and review to evaluate cases of concomitant IBD and thyroid diseases.

\section{Methods}

We performed a review of the English and Japanese literature regarding the coexistence of thyroid diseases and IBD. We used PubMed and Japana Centra Revuo Medicina (Igaku Chuo Zasshi) for the English and Japanese literature, respectively.

According to the evaluation of thyroid dysfunction, hyperthyroidism is diagnosed when both the serum-free triiodothyronine (fT3) and free thyroxin (fT4) levels are elevated, but the thyroid stimulating hormone (TSH) levels are restrained. Hypothyroidism is diagnosed when serum levels of both fT3 and fT4 are decreased, but the level of TSH is elevated. Moreover, subclinical hyperthyroidism 
TABLE 1: The reported prevalence of hyperthyroidism in inflammatory bowel disease.

\begin{tabular}{lcccc}
\hline Authors (year) & Type of IBD & Prevalence in IBD & Prevalence in general populations & Reference \\
\hline Järnerot et al. (1975) & UC & $3.7 \%(11 / 300)^{*}$ & $0.83 \%(5 / 600)^{*}$ & $0.67 \%(2 / 300)$ \\
Snook et al. (1989) & UC & $1.5 \%(13 / 858)$ & $0.67 \%(2 / 300)$ & {$[16]$} \\
& CD & $0.26 \%(1 / 378)$ & $0 \%(0 / 206)$ & {$[15]$} \\
Pooran et al. (2003) & CD & $0.95 \%(2 / 210)$ & $0 \%(0 / 66)$ & {$[15]$} \\
Casella et al. (2008) & UC & $0.62 \%(1 / 162)$ & $0 \%(0 / 66)$ & {$[24]$} \\
Yakut et al. (2011) & UC & $4.4 \%(5 / 113)$ & - & {$[24]$} \\
Liu et al. (2013) & CD & $0 \%(0 / 33)$ & $0 \%(0 / 44)$ & {$[19]$} \\
\hline
\end{tabular}

IBD: inflammatory bowel disease; UC: ulcerative colitis; CD: Crohn's disease.

${ }^{*}$ The prevalence was significantly higher in UC patients than in the general populations $(p<0.01)$.

TABLE 2: The reported prevalence of hypothyroidism in inflammatory bowel disease.

\begin{tabular}{lcccc}
\hline Authors (year) & Type of IBD & Prevalence in IBD & Prevalence in general populations & Reference \\
\hline Snook et al. (1989) & UC & $0.93 \%(8 / 858)$ & $0.67 \%(2 / 300)$ & $0.67 \%(2 / 300)$ \\
& CD & $0.53 \%(2 / 378)$ & $8.3 \%(17 / 206)^{*}$ & {$[15]$} \\
Pooran et al. (2003) & CD & $3.8 \%(8 / 210)^{*}$ & $6.3 \%(360 / 5721)^{*}$ & {$[23]$} \\
Casella et al. (2008) & UC & $1.9 \%(3 / 162)^{*}$ & $0 \%(0 / 66)$ & {$[5]$} \\
Yakut et al. (2011) & CD & $0 \%(0 / 33)$ & - & {$[19]$} \\
Liu et al. (2013) & CD & $2.3 \%(1 / 44)$ & -
\end{tabular}

IBD: inflammatory bowel disease; UC: ulcerative colitis; CD: Crohn's disease.

${ }^{*}$ The prevalence was significantly higher in the general populations than in IBD patients.

is diagnosed when serum levels of both fT3 and fT4 are within the normal range, but the level of TSH is decreased. Furthermore, subclinical hypothyroidism is diagnosed when serum levels of both fT3 and fT 4 are within the normal range, but the level of TSH is elevated.

\section{Prevalence of Concomitant Thyroid Dysfunction in IBD}

3.1. Prevalence of Thyroid Dysfunction in General Populations. The studies identified by the literature search indicated a $2 \%-8 \%$ prevalence of thyroid dysfunction (hyper- or hypothyroidism) in the general population, including the populations in iodine-deficient countries [5]. The prevalence of hyperthyroidism in women is between $0.5 \%$ and $2 \%$ and it is 10 times less common in men [6]. The incidence and prevalence of overt hypothyroidism have been reported to be $2-4 / 1,000$ population/year and $0.8 \%-1.3 \%$, respectively [7].

3.2. Prevalence of Concomitant Thyroid Dysfunction in UC. Some studies have reported a similar prevalence of thyroid dysfunction in UC patients $(2.2 \%-8.0 \%)$ compared with general populations [5, 8-12]. Moreover, Casella et al. [5] found that the prevalence of thyroid dysfunction in the general population in Italy was $7.5 \%$ (429/5721), which was significantly higher than that in UC patients $(2.5 \%$; 4/162). On the other hand, some population studies have demonstrated a two- to fourfold increase in the prevalence of thyroid dysfunction in patients with UC compared with the prevalence in the general population [13-16].
The reported prevalence of hyperthyroidism in UC patients was $0.62 \%-3.7 \%[3,5,15,17-21]$. On the other hand, the prevalence of UC in patients with hyperthyroidism was $1.34 \%$ [22]. Casella et al. [5] also reported that the prevalence of hyperthyroidism in the general population in Italy was $1.21 \%(69 / 5721)$ and was $0.62 \%(1 / 162)$ in UC patients. Snook et al. [15] reported that the prevalence of hyper- and hypothyroidism in UC patients was $1.5 \%$ and $0.9 \%$, respectively; however, the prevalence of hyper- and hypothyroidism in the control group was $0.7 \%$ for both [15]. Based on the findings of their study, the development of autoimmune disorders, including thyroid diseases, did not show a clear temporal relationship with the onset or activity of IBD, with the exception of concomitant cases of autoimmune hemolytic anemia in UC patients [15]. In addition, a study by Järnerot et al. [16] reported that the prevalence of hyperthyroidism in UC patients was significantly higher compared with that in controls (3.7\% versus $0.8 \% ; p<0.01)$. Recently, there have been few studies that have specifically investigated cases of concomitant hyperthyroidism and UC. These studies are summarized in Tables 1 and 2.

3.3. Prevalence of Concomitant Thyroid Dysfunction in CD. Some studies reported that there were no significant differences in thyroid function test (serum levels of fT3, fT4, and TSH) between $\mathrm{CD}$ patients and controls [24, 51]. In a study by Snook et al. [15], the prevalence of hyperand hypothyroidism in CD patients was $0.3 \%$ and $0.5 \%$, respectively. In the same study, the prevalence of hyperand hypothyroidism in the control group was $0.7 \%$ for both [15]. Yakut et al. [24] reported that the prevalence of both 
TABLE 3: Characteristics of the patients with concomitant ulcerative colitis and Graves' disease.

\begin{tabular}{|c|c|c|c|c|c|c|c|c|}
\hline Case & (Year) & Gender & $\begin{array}{c}\text { Age at } \\
\text { diagnosis of } \\
\text { UC (years) }\end{array}$ & $\begin{array}{c}\text { Age at } \\
\text { diagnosis of } \\
\text { GD (years) }\end{array}$ & $\begin{array}{l}\text { UC prior } \\
\text { to GD }\end{array}$ & UC type & Complications & Reference \\
\hline 1 & $(1980)$ & $\mathrm{F}$ & 46 or 47 & 46 & - & Left-sided colitis? & & {$[18]$} \\
\hline 2 & $(1980)$ & $\mathrm{M}$ & 18 or 19 & 18 & - & Pancolitis? & & {$[18]$} \\
\hline 3 & $(1981)^{*}$ & $\mathrm{~F}$ & 53 & 53 or 54 & + & ? & Dermatomyositis & [25] \\
\hline 4 & $(1984)^{*}$ & M & 46 & 36 & - & Pancolitis & & {$[9]$} \\
\hline 5 & $(1985)^{*}$ & $\mathrm{~F}$ & 66 & 46 & - & Pancolitis & & [26] \\
\hline 6 & $(1996)^{*}$ & $\mathrm{~F}$ & $31 ?$ & 30 & - & Left-sided colitis & & {$[10]$} \\
\hline 7 & (1998) & M & 17 & 24 & + & ? & Primary sclerosing cholangitis & [27] \\
\hline 8 & $(1999)^{*}$ & $\mathrm{~F}$ & 32 & 30 & - & $?$ & & {$[11]$} \\
\hline 9 & $(2001)$ & $\mathrm{F}$ & 41 & 41 & Sim & $?$ & & {$[13]$} \\
\hline 10 & $(2001)$ & M & 14 & 26 & + & Pancolitis? & & {$[14]$} \\
\hline 11 & $(2001)^{*}$ & $\mathrm{~F}$ & 35 & $31 ?$ & - & Left-sided colitis & Familial GD & {$[28]$} \\
\hline 12 & $(2001)^{*}$ & M & 24 & 26 & + & Pancolitis & & [8] \\
\hline 13 & (2005) & $\mathrm{F}$ & 42 & 47 & + & ? & & [17] \\
\hline 14 & (2008) & $\mathrm{F}$ & 61 & 60 & - & $?$ & & [5] \\
\hline 15 & $(2009)^{*}$ & M & 22 & 26 & + & Pancolitis & Familial UC & {$[29]$} \\
\hline 16 & $(2012)$ & $\mathrm{F}$ & 38 & 18 & - & ? & IgA nephropathy & [30] \\
\hline
\end{tabular}

UC: ulcerative colitis; GD: Graves' disease; F: female; M: male; Sim: simultaneous.

*Japanese-language literature.

hyper- and hypothyroidism in patients with $\mathrm{CD}$ as well as for both control groups was $0 \%(0 / 33)$ and $0 \%(0 / 66)$, respectively. Liu et al. [19] reported that the prevalence of hyper- and hypothyroidism in patients with $\mathrm{CD}$ was $0 \%$ $(0 / 44)$ and $2.3 \%(1 / 44)$, respectively. In a study by Pooran et al. [23], the prevalence of hypothyroidism was lower in CD patients $[3.8 \%(8 / 210)]$ than in control individuals $[8.2 \%$ (17/206)], although the prevalence of hyperthyroidism was statistically similar between the groups. These studies are summarized in Tables 1 and 2 .

\section{Concomitant GD in IBD}

4.1. GD. GD, also known as Basedow's disease in Europe, is the most common cause of hyperthyroidism [3,29]. It is one of the most common autoimmune disorders, with an annual incidence of approximately 14 individuals per 100,000 [52]. GD is caused by circulating antibodies (anti-TSH receptor autoantibodies) that mimic the actions of TSH, resulting in the increased synthesis and release of thyroid hormones [52]. GD is associated with extrathyroidal manifestations, including orbital disease (ophthalmopathy), skin changes, and sometimes fingertip and nail abnormalities [53]. The diagnostic criteria for GD include clinical and/or biochemical evidence of thyrotoxicosis and one or more of the following features: (1) the presence of serum TSH receptor autoantibodies (TRAbs), (2) ophthalmopathy and/or dermopathy, and (3) diffuse elevated thyroid radioiodine uptake [52].

4.2. Concomitant GD and UC. In general, the pathophysiology of UC is associated with the Th2 cytokine phenotype, and there is increased Th2 activity in GD [21]. Therefore, both
GD and UC are associated with a Th1/Th2 imbalance, with a dominance of $T h 2$ responses [13, 21, 22, 28, 29]. Matsumura et al. [21] reported on a 26-year-old female who had a flare-up of UC and hyperthyroidism that was successfully treated with infliximab. In addition, they reported that the Th1/Th2 imbalance was improved 2 weeks after the initiation of infliximab therapy. However, it is still unclear whether GD is an extraintestinal manifestation of UC or not [17]. In addition, some studies have found no differences in the prevalence of hyperthyroidism between UC patients and the general population [5]. Therefore, it is currently unclear whether concomitant GD and UC occur by chance or reflect a common immunological basis [8].

One accepted hypothesis for the pathogenesis of IBD is that the mucosal immune system exhibits an aberrant response toward luminal antigens such as commensal bacteria [54]. For example, a chronic low-grade portal infection, caused by UC and leading to chronic biliary tract inflammation and fibrosis, has been suggested as a pathogenic mechanism [55]. However, because the colon and thyroid do not have the same embryological origins, the same trigger antibodies may not be the cause of the association between GD and UC $[13,21]$.

According to Casella et al. [5], the first case of concomitant hyperthyroidism and UC was reported in 1968. In total, we identified 16 reported cases of concomitant GD and UC (eight in the English-language literature $[5,13,14,17,18,27$, $30]$ and eight in the Japanese-language literature [8-11, 25, $26,28,29])$. The characteristics of these 16 reported cases of concomitant GD and UC are summarized in Table 3 . We excluded the cases of concomitant UC and hyperthyroidism that were caused by excessive supplementation of thyroxine 
TABLE 4: Characteristics of the patients with concomitant Crohn's disease and Graves' disease.

\begin{tabular}{|c|c|c|c|c|c|c|c|c|c|c|}
\hline \multirow[b]{2}{*}{ Case } & \multirow[b]{2}{*}{ (Year) } & \multirow[b]{2}{*}{ Gender } & \multirow{2}{*}{$\begin{array}{l}\text { Age at diagnosis } \\
\text { of CD (years) }\end{array}$} & \multirow{2}{*}{$\begin{array}{l}\text { Age at diagnosis } \\
\text { of GD (years) }\end{array}$} & \multirow{2}{*}{$\begin{array}{l}\text { CD prior to } \\
\text { GD }\end{array}$} & \multicolumn{3}{|c|}{ Thyroid function tests } & \multirow[b]{2}{*}{ Remarks } & \multirow[b]{2}{*}{ Reference } \\
\hline & & & & & & $\begin{array}{c}\mathrm{TSH} \\
(\mu \mathrm{U} / \mathrm{mL})\end{array}$ & $\begin{array}{c}\text { freeT3 } \\
(\mathrm{pg} / \mathrm{mL})\end{array}$ & $\begin{array}{l}\text { freeT4 } \\
(\mathrm{ng} / \mathrm{dL})\end{array}$ & & \\
\hline \multirow[t]{2}{*}{1} & (1999) & M & 14 & 14 & Sim & $<0.1$ & $?$ & $?$ & & {$[31]$} \\
\hline & & & & & & & $\rightarrow$ norn & lization & & \\
\hline \multirow[t]{2}{*}{2} & $(2004)^{*}$ & M & 19 or 20 & 20 & + & 0.05 & 5.6 & 3.7 & & {$[32]$} \\
\hline & & & & & & & $\rightarrow$ norn & lization & & \\
\hline \multirow[t]{2}{*}{3} & $(2005)$ & F & 22 & 38 & + & 0.017 & $>20$ & $>12$ & Familial GD & {$[22]$} \\
\hline & & & & & & & $\rightarrow$ norn & lization & $\mathrm{CD} \rightarrow$ ileotomy & \\
\hline
\end{tabular}

CD: Crohn's disease; GD: Graves' disease; F: female; M: male; Sim: simultaneous.

* Japanese-language literature.

after a subtotal thyroidectomy for toxic adenoma, by toxic multinodular goiter, or by unknown causes [56]. Some reports discussed cases of UC induced or aggravated by the administration of rituximab used to treat GD. Therefore, we also excluded the suspected rituximab-induced UC cases with GD $[57,58]$ from these concomitant UC and GD cases.

Hyperthyroidism is more common in females than in males, with a female-to-male ratio of $10: 1[6,29]$. In contrast, $\mathrm{UC}$ is not a gender-specific disease; the female-to-male ratio for UC ranges from 0.51 to 1.58 [59]. Of the 16 cases of concomitant GD and UC that were identified in this review, 6 (37.5\%) were male and $10(62.5 \%)$ were female. In most cases of concomitant GD and UC that had been reported in the 1980s and 1990s, GD was diagnosed prior to the development of UC. However, in the cases reported in the 2000s, there was no clear tendency in the order of diagnosis. In fact, of the 16 cases reported since 1980, UC developed before GD in six cases (37.5\%). In nine cases (56.3\%), GD developed before UC, and both UC and CD were simultaneously diagnosed in one case $(6.3 \%)$. The diagnosis of the concomitant disease occurred between the ages of 18/19 and 61 years, and the time interval between the diagnosis of the primary and concomitant disease was 0-20 years. The types of UC in the 16 cases of concomitant GD and UC included 6 cases of pancolitis and 3 cases of left-sided colitis; there were no cases of proctitis. The type of UC was unclear in seven cases (Table 3 ).

There were no reports of a flare-up of UC soon after the onset of GD. In most cases of concomitant GD and UC, UC was treated with medications such as aminosalicylates and corticosteroids. Only three of these cases required surgery (colectomy) for persistent colitis despite pharmacotherapy $[5,14,29]$. There were no reports of severe complications of UC such as toxic megacolon in these cases. In most cases, the GD was treated with antithyroid agents and only one case required surgery (subtotal thyroidectomy) [17]. There were no reports of death related to concomitant GD and UC and no evidence that patients with concomitant GD and UC had a poorer prognosis than those with UC but not GD.

\subsection{Concomitant $G D$ and $C D$}

4.3.1. Genetic Associations between GD and CD. Some studies have assessed possible common genetic factors between GD and CD. The role of non-HLA genes such as PTPN22,
CTLA4, and CD40 in GD patients has been extensively investigated [60]. Although some studies have reported that PTPN22 did not influence the risk of IBD, including CD [61], other studies have reported that PTPN22 may influence the risk of developing CD $[62,63]$. Moreover, some studies have also reported that CTLA4 may influence the risk of developing CD $[64,65]$. In a Spanish meta-analysis, the frequency of the minor allele rs1883832T on the CD40 gene was significantly higher in CD patients than in control individuals, but it was not significantly higher in UC patients [66]. However, further investigations may be necessary to identify any common genetic factors responsible for CD and GD. At present, it is uncertain whether cases of concomitant $\mathrm{CD}$ and GD occur due to common genetic backgrounds.

4.3.2. Characteristics of Cases of Concomitant GD and CD. The characteristics of the 3 reported cases of concomitant CD and GD are summarized in Table 4 [22, 31, 32]. Of the three cases of concomitant CD and GD that were identified in this review, two cases were male and one was female. In two cases, CD was diagnosed before the development of GD. The diagnosis of the concomitant diseases was made between the ages of 14 and 38 years, and the interval between the diagnoses of the primary and concomitant diseases was 0-16 years. Only 1 case required surgery (ileotomy) for perforation of the ileum, which had occurred 22 years before the development of GD [22] (Table 4). In all three cases, thyroid dysfunctions were normal after pharmacotherapy. There were no deaths due to CD or GD.

On the other hand, Kettaneh et al. reported on two cases of hyperthyroidism that were caused by adenomatous goiter or Plummer's disease in CD and Takayasu arteritis [56].

\section{HT in IBD}

5.1. HT. HT, which is also known as autoimmune thyroiditis, is one of the most common autoimmune endocrine diseases, characterized by an autoimmune-mediated destruction of the thyroid gland and predominantly affecting women [67]. HT may also be characterized by an enlarged thyroid gland and is identified histologically by lymphocytic thyroid infiltration and positive antibody tests for antithyroglobulin and/or antithyroid peroxidase antibodies [4, 67]. HT is a common cause of hypothyroidism. However, some cases 
exhibit normal thyroid hormone (including TSH) levels, whereas other cases exhibit subclinical hypothyroidism in the presence of increased TSH levels. HT can also cause hyperthyroidism due to destructive (painless) thyroiditis [67]. The diagnosis of HT relies on the demonstration of circulating antibodies to thyroid antigens (mainly thyroperoxidase and thyroglobulin) and reduced echogenicity on the sonography of patients with proper clinical features. The treatment is based on the administration of synthetic thyroid hormones to correct for hypothyroidism [67].

5.2. Prevalence of Concomitant HT in IBD. In Japan, the prevalence of HT (chronic thyroiditis) in UC patients was reported as $0.14 \%(8 / 5833)$ during the 1980 s and as $0.07 \%$ $(1 / 1433)$ during the 1990s $[8,11]$. Bardella et al. [68] reported that the prevalence of HT in UC patients was $2.2 \%(2 / 90)$ and that of HT in CD patients was 4.4\% (4/90). Yakut et al. [24] reported that the prevalence of HT in UC patients was 3.5\% $(4 / 113)$ and that HT in CD patients was 0\% (0/33). Cesarini et al. [20] also reported that the prevalence of HT in UC patients was $1.8 \%(8)$ and that HT in CD patients was $2.2 \%(10 / 464)$ in Italy. In a large population-based study in Canada that included 8072 IBD patients (3879 UC and 4193 CD patients), the prevalence of HT (autoimmune thyroiditis) was similar to that of the controls [69].

5.3. Concomitant Cases of HT and UC. Although sporadic cases of concomitant HT and UC have been reported, most reports were cases of autoimmune diseases such as immune thrombocytopenic purpura, type 1 diabetes mellitus, or autoimmune hepatitis other than HT that had also occurred in patients with UC and HT [70, 71]. Moreover, ATDs are one of the components in the multiple autoimmune syndrome, which consists of three or more well-defined autoimmune conditions in the same patient [72]. Moreover, some authors have reported a case with concomitant UC and HT in a patient with Turner syndrome [73, 74]. In a cohort study of 2,459 patients, the risk of concomitant HT and IBD (both UC and CD) was significantly higher in Turner syndrome patients compared with the general population [75]. This suggests that $\mathrm{HT}$ and $\mathrm{UC}$ can be a manifestation of Turner syndrome.

5.4. Concomitant Cases of HT and CD. The characteristics of the 10 previously reported cases of concomitant $\mathrm{CD}$ and HT are summarized in Table 5 (eight in the English-language literature $[22,33,34,36,38,39]$ and two in the Japaneselanguage literature $[35,37])$. Of these, three cases occurred in males and seven occurred in females. After excluding three cases that were simultaneously diagnosed with $\mathrm{CD}$ and HT, $\mathrm{CD}$ was diagnosed before the development of HT in five of the remaining seven cases. The concomitant disease was diagnosed between the ages of 10 and 55 years, and the interval between the diagnoses of the primary and concomitant disease was 0-27 years. Regarding thyroid function, there were four cases of hypothyroidism [33,35,37], four cases of hyperthyroidism due to suspected destructive (or painless) thyroiditis $[22,36,38,39]$, and one case with normal function [33]; the thyroid function was unclear in one case because the results of thyroid function test (fT3/fT4 and TSH levels) were not mentioned in that paper [34]. The treatment included administration of synthetic thyroid hormones in cases with hypothyroidism. Moreover, no thyroid dysfunction cases resistant to treatments were observed. There were no deaths due to $\mathrm{CD}$ or $\mathrm{HT}$. One of the 10 cases represented familial CD [39].

Among the reported cases, Noto et al. [35] described a case of a young female who was diagnosed almost simultaneously with CD and HT; she also had Turner syndrome. As previously discussed, the risk of concomitant HT and IBD (both UC and CD) was significantly higher in Turner syndrome patients compared with the general population [75].

\section{SAT in IBD}

SAT is an uncommon condition, yet it is considered as the most common cause of painful thyroiditis. The disease is believed to have a viral origin, although the precise etiology of SAT is unknown. Common laboratory findings include transient thyrotoxic conditions and poor or no thyroid uptake. Within a few weeks and after the depletion of any preformed thyroid hormone, approximately $30 \%$ of patients will undergo a hypothyroid phase [76]. Standard treatment for SAT is administration of prednisolone. Moreover, SAT-like syndrome (symptoms) may have similar clinical symptoms as SAT and the thyroid function in SAT-like syndrome is within the normal range [41, 50,77]. The development of SAT in IBD patients appears to be extremely rare. A few cases of SAT or SAT-like syndrome have occurred in IBD patients $[50,77,78]$. One case report of SAT in IBD was reported by Horai et al. [78]. They reported on a female UC patient who was diagnosed with SAT and Takayasu's arteritis $>20$ years after the diagnosis of UC. This suggests that genetic factors may be associated with the occurrence of these conditions [78]. In another case, Kawashima et al. [50] reported a middle-aged man with $A G$ and $C D$, who developed hyperthyroidism caused by SAT after the administration of the tumor necrosis factor- (TNF-) $\alpha$ inhibitor infliximab, and subsequent SAT-like symptoms then developed after the administration of another TNF- $\alpha$ inhibitor, adalimumab (suspected drug-induced thyroiditis). However, after an examination of 36 rheumatic patients, Kaklamanos et al. [79] reported that infliximab and rituximab did not cause any alterations in thyroid function and/or autoimmunity (thyroid antibody titer), even in patients with previously undiagnosed autoimmune thyroid diseases. Ikenoue et al. [77] described recurrent SAT-like symptoms in two cases of IBD (one with $\mathrm{CD}$ and the other with suspected indeterminate colitis) that were complicated by thyroid amyloidosis. Therefore, SAT or SAT-like syndrome should be considered in IBD patients that have complications such as amyloid deposition in the thyroid. Moreover, in these cases, SAT or SAT-like syndromes resolved after prednisolone treatment.

\section{AG in IBD}

7.1. Amyloidosis in IBD. Amyloidosis is characterized by the deposition of fibrillary proteins that can accumulate in various organs [49] and is classified as primary or secondary. 


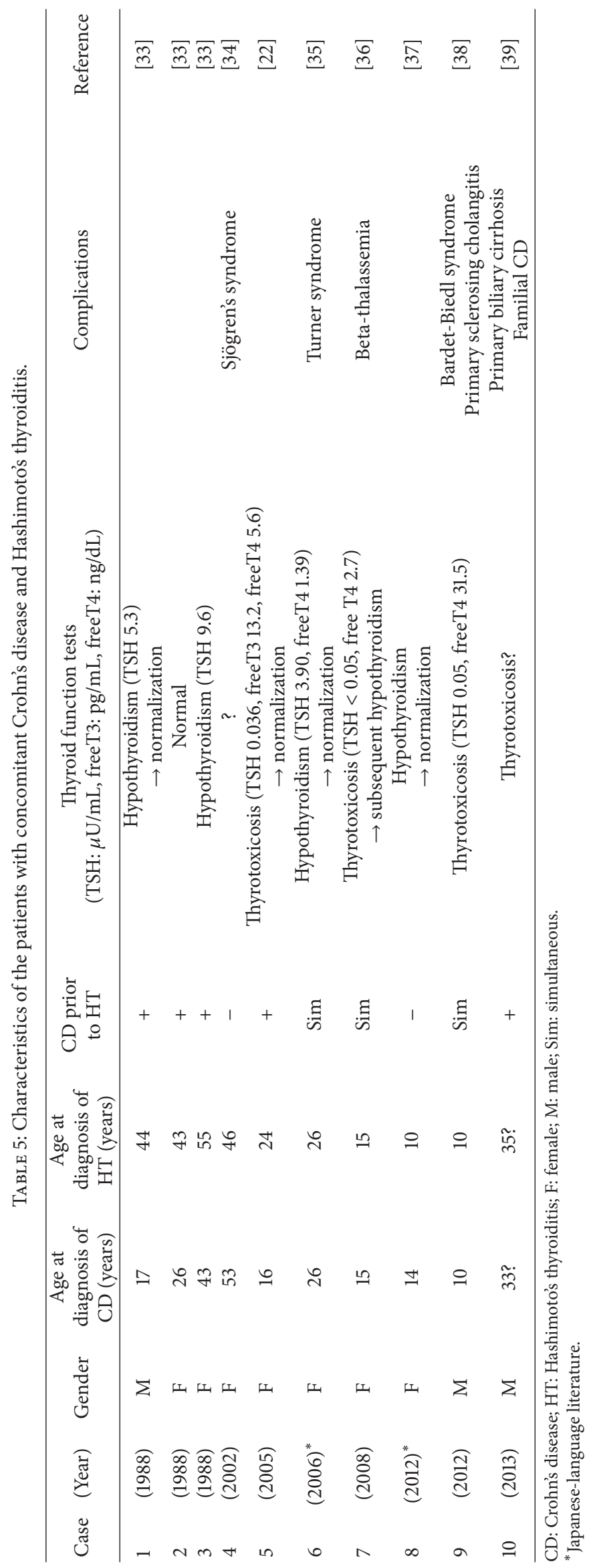


Secondary amyloidosis is a well-known complication of CD, as demonstrated by previous studies, and has an incidence of $0.5 \%-9.0 \%[42,48]$. However, the prevalence of amyloidosis is less in UC patients. Greenstein et al. [40] reported on 16 cases $(0.52 \% ; 15$ of $\mathrm{CD}$ and 1 of UC) among 3,050 IBD cases and found that the prevalence of amyloidosis was $0.88 \%$ $(15 / 1709)$ in CD patients and was $0.07 \%$ (1/1341) in UC patients. They also found that only one case $(0.03 \%$ of all IBD patients and $0.06 \%$ of all CD patients) had AG. Moreover, they reviewed 25 IBD cases ( 22 of CD and 3 of UC) that were complicated with amyloidosis and reported that renal disturbances occurred in $84 \%(21 / 25)$ of IBD patients; most deaths in this population were associated with renal complications such as nephrotic syndrome or renal failure [40].

7.2. $A G$ in IBD. AG, characterized by thyroid enlargement caused by extensive amyloid deposition in the thyroid gland $[40,49,80]$, is an uncommon complication of IBD. In most cases, AG is associated with amyloid deposition in other organs during the course of systemic amyloidosis and with the glandular deposition of amyloid AA [81]. AG is clinically characterized by the rapid growth of the thyroid gland, resulting in the development of pressure symptoms [80]. Although thyroid function in AG patients is usually within the normal range, hyper- and hypothyroidism develop sometime [42, 49, 80, 81]. However, Kimura et al. [82] reported that 9 of 10 (90\%) AG patients had abnormal thyroid function, including the low T3 syndrome; they also found that five patients had hypothyroidism, one had hyperthyroidism, one had transient hypothyroidism, and two had low T3 syndrome.

7.3. AG in UC. Although sporadic cases of CD complicated with AG have been reported, the development of AG in UC patients appears to be extremely rare. To the best of our knowledge, there have been no reports of such concomitant cases in the English literature. However, one such case was discussed in a Japanese proceeding that involved a 35-yearold female with UC and renal amyloidosis (chronic renal failure on hemodialysis) who had developed AG with a slight tendency of hypothyroidism [83]. However, AG did not result in the flare-up of UC.

7.4. $A G$ in $C D$. Our literature review identified 12 reports (seven in English-language [40, 42, 47-50], one in Spanish [46], and four in Japanese-language [41, 43-45]) of CD complicated with AG, which are summarized in Table 6. The cases of CD with focal amyloid deposition and without goiter were excluded from our review. Among these cases, seven occurred in males and four in females; the sex of one patient was unclear [47]. In these cases, AG was diagnosed between the ages of 26 and 58 years. The major clinical symptoms were the development of a painful neck mass and associated swelling. With regard to thyroid function in the 11 cases (excluding the one case in which the data was not available [40]), the thyroid function in eight was within the normal range [41-43, 46-50], two were diagnosed with hypothyroidism $[45,48]$, and one was diagnosed with subclinical hypothyroidism [44]. These AG cases were diagnosed by histopathological findings and/or Congo red staining after thyroidectomy or fine-needle aspiration biopsy. Among these 12 cases, one case report suggested that the development of goiter was associated with the activity of CD [41]. However, there were no obvious correlations between the development of AG and activity or flare-up of IBD; four cases showed no correlations between the development of goiter and activity or flare-up of $\operatorname{CD}[42,44,48,50]$ and these correlations were not mentioned in the remaining seven cases. Standard treatment including diagnosis for AG in concomitant CD and AG cases was surgery (thyroidectomy).

Among these 12 cases, three were complicated with chronic renal failure due to renal amyloidosis $[40,43,44]$ and two others were complicated with nephrotic syndrome due to renal amyloidosis $[46,48]$. Outcomes of AG in CD patients may be partially dependent on other organ disturbances by amyloid deposition, such as renal failure. Only one death occurred due to renal failure associated with renal amyloidosis [40].

\section{Thyroid Cancer in IBD}

Thyroid cancer includes papillary thyroid cancer (PTC), follicular cancer, medullary cancer, and poorly or undifferentiated differentiated cancer. The case reports of concomitant cases of thyroid cancer [84-89] or primary thyroid lymphoma $[90,91]$ and IBD have been sporadic. There have been case reports of PTC in patients with UC $[85,86,88]$ and CD $[84,87,89]$. Although primary lymphoma of the thyroid is quite rare, its prevalence is increasing in a proportion of cases with HT. Further, Triantafillidis [90] reported a case with concomitant primary thyroid lymphoma and HT in a patient with UC.

Moss et al. [84] reported five cases of PTC in CD patients. Among these cases, one patient had been on immunomodulator therapy before the diagnosis of thyroid cancer. They suspected that exposure to radiation through imaging (computed tomography or small bowel radiological investigations) in early adulthood or while receiving multivitamins might have been the cause [84]. However, none of the patients had a history of thyroid dysfunction, radiation exposure, or family history of thyroid cancer [84]. Radiation exposure at a young age has been a consistent risk factor for PTC, and the risk of PTC is higher in women with a high multivitamin supplement intake $[84,92]$.

Yano et al. [93] demonstrated that the incidence of thyroid cancer in 770 Japanese CD patients was not significantly higher compared with healthy populations. Moreover, they found that previous 11 studies demonstrated that the incidence of thyroid cancer in CD patients was not significantly higher than that in healthy populations [93]. Sonu et al. [94] suggested that the age at diagnosis of PTC in CD patients was significantly lower than that in UC patients or control populations and that patients with CD and UC were not less likely to develop PTC compared to those control populations in the United States.

\section{Nonthyroidal Illness Syndrome in IBD}

Nonthyroidal illness syndrome (NTIS) is characterized as a significantly low serum level of fT3 in patients with 


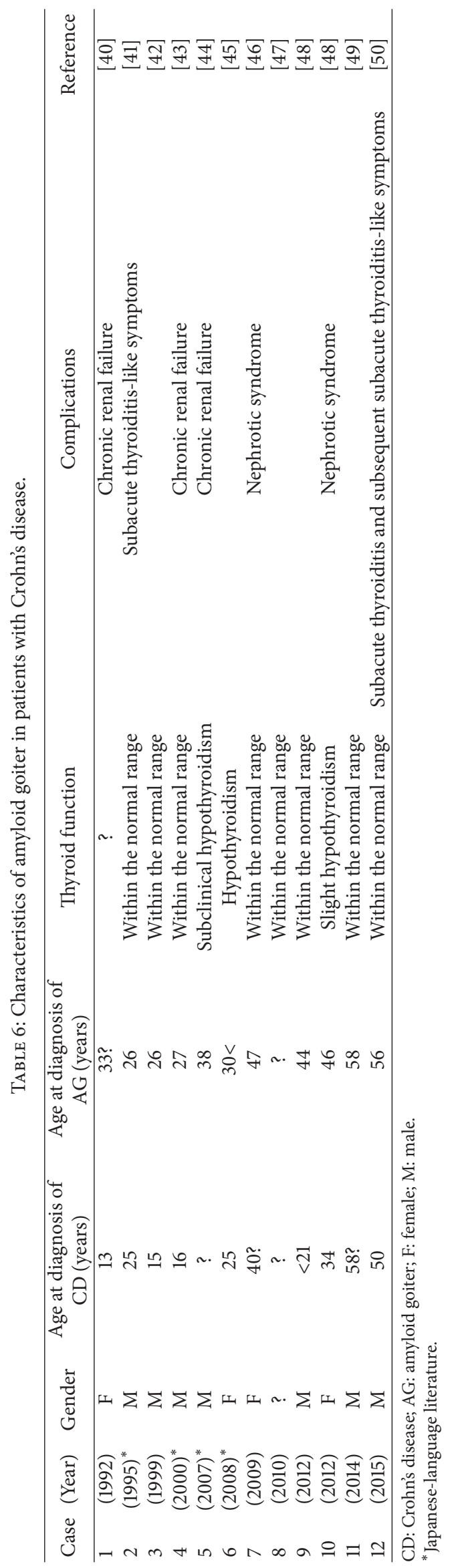


nonthyroidal diseases, such as various acute and chronic diseases [19]. Although there were few studies on NTIS in IBD patients, Liu et al. [19] reported that the prevalence of NTIS in CD patients was relatively common [36.4\% (16/44)], and CD patients with NTIS displayed worse nutritional status and clinical outcome. Moreover, compared with CD patients with euthyroidism, these patients also exhibited enhanced critical disease activity and severity.

\section{Ectopic Thyroid Gland in IBD}

An ectopic thyroid gland is a rare congenital abnormality; this condition is caused by the failure of the gland anlage to descend early in the course of embryogenesis. Research has estimated its frequency to be $1 / 4000$ to $1 / 8000$ among patients with hypothyroidism and to be $0.3 \%$ among all thyroid diseases [95]. To the best of our knowledge, there have been no cases of concomitant ectopic thyroid gland and IBD.

\section{Conclusions}

In this report, we conducted a literature search and review to evaluate the cases of concomitant IBD and thyroid diseases or thyroid dysfunction, ATDs (GD and HT), SAT, AG, and thyroid cancer. At present, there might be no obvious differences in the prevalence of thyroid dysfunction, GD, and thyroid cancer between IBD patients and the general population. However, concomitant UC and HT might be relatively common in particular patients with multiple autoimmune disorders. Moreover, there might be no correlation between development of thyroid disease and severity of IBD. There might be no obvious differences of fatal prognoses between patients with concomitant thyroid diseases and IBD and patients with thyroid diseases without IBD. However, it will be necessary to gather additional data to clarify these conditions.

\section{Conflict of Interests}

There is no conflict of interests to declare.

\section{Acknowledgment}

The author would like to thank the members of his department.

\section{References}

[1] M. Fakhoury, R. Negrulj, A. Mooranian, and H. Al-Salami, "Inflammatory bowel disease: clinical aspects and treatments," Journal of Inflammation Research, vol. 7, no. 1, pp. 113-120, 2014.

[2] J. K. Triantafillidis, C. A. Manoussakis, C. Tsafaras, and A. Koutsorizof, "Coexistence of thyreotoxicosis and exacerbation of ulcerative colitis," The American Journal of Gastroenterology, vol. 85, no. 7, pp. 908-910, 1990.

[3] J. K. Triantafillidis, A. Cherakakis, and M. Theororou, "Coexistence of hyperthyroidism and ulcerative colitis: report of 4 cases and a review of the literature," Italian Journal of Gastroenterology, vol. 24, no. 9, pp. 494-497, 1992.
[4] G. Radetti, "Clinical aspects of Hashimoto's thyroiditis," Endocrine Development, vol. 26, pp. 158-170, 2014.

[5] G. Casella, E. De Marco, E. Antonelli et al., "The prevalence of hyper- and hypothyroidism in patients with ulcerative colitis," Journal of Crohn's and Colitis, vol. 2, no. 4, pp. 327-330, 2008.

[6] A. Gessl, R. Lemmens-Gruber, and A. Kautzky-Willer, "Thyroid disorders," in Sex and Gender Differences in Pharmacology, vol. 214 of Handbook of Experimental Pharmacology, pp. 361-386, Springer, Berlin, Germany, 2012.

[7] G. H. Seo and J. H. Chung, "Incidence and prevalence of overt hypothyroidism and causative diseases in Korea as determined using claims data provided by the health insurance review and assessment service," Endocrinology and Metabolism, vol. 30, no. 3, pp. 288-296, 2015.

[8] M. Tomonaga, K. Nakamura, H. Kinoshita et al., "A case of ulcerative colitis associated with Graves' disease," Japanese Journal of Gastroenterology, vol. 98, no. 6, pp. 644-649, 2001.

[9] M. Itoh, M. Funauchi, N. Fukuma et al., "A case of hyperthyroidism complicated with ulcerative colitis," Saishin Igaku, vol. 39, pp. 167-171, 1984.

[10] T. Nishi, T. Yanagawa, K. Machida, K. Okai, K. Naka et al., "A case of Basedow's disease complicated with ulcerative colitis," Hormone to Rinsho, vol. 44, pp. 109-111, 1996.

[11] K. Okai, K. Machida, M. Nishi, and K. Nanjo, "Complications of extraintestinal endocrine disease associated with ulcerative colitis-association of ulcerative colitis and autoimmune thyroid disease," Nihon Rinsho, vol. 57, no. 11, pp. 2536-2539, 1999.

[12] G. Tomasello, P. Tralongo, F. Amoroso et al., "Dysmicrobism, inflammatory bowel disease and thyroiditis: analysis of the literature," Journal of Biological Regulators \& Homeostatic Agents, vol. 29, no. 2, pp. 265-272, 2015.

[13] E. S. Bonapace and R. Srinivasan, "Simultaneous occurrence of inflammatory bowel disease and thyroid disease," American Journal of Gastroenterology, vol. 96, no. 6, pp. 1925-1926, 2001.

[14] M. Nishimura, T. Yamamoto, H. Iijima, Y. Moriwaki, S. Takahashi, and T. Hada, "Basedow's disease and chronic ulcerative colitis: a case report and review of the Japanese literature," Internal Medicine, vol. 40, no. 1, pp. 44-47, 2001.

[15] J. A. Snook, H. J. de Silva, and D. P. Jewell, "The association of autoimmune disorders with inflammatory Bowel disease," The Quarterly Journal of Medicine, vol. 72, no. 269, pp. 835-840, 1989.

[16] G. Järnerot, A. K. Azad Khan, and S. C. Truelove, "The thyroid in ulverative colitis and Crohn's disease. II. Thyroid enlargement and hyperthyroidism in ulcerative colitis," Acta Medica Scandinavica, vol. 197, no. 1-2, pp. 83-87, 1975.

[17] T. Iiai, T. Honma, T. Ishikawa, H. Shimizu, and K. Hatakeyama, "Hyperthyroidism in association with ulcerative colitis: a case report," Acta Medica et Biologica, vol. 53, no. 2, pp. 61-64, 2005.

[18] S. K. Iyer and R. G. Karlstadt, "Hyperthyroidism and ulcerative colitis: report of two cases and a review of the literature," Journal of the National Medical Association, vol. 72, no. 2, pp. 127-131, 1980.

[19] S. Liu, J. Ren, Y. Zhao et al., "Nonthyroidal illness syndrome: is it far away from crohn's disease?" Journal of Clinical Gastroenterology, vol. 47, no. 2, pp. 153-159, 2013.

[20] M. Cesarini, E. Angelucci, M. Rivera et al., "Thyroid disorders and inflammatory bowel diseases: retrospective evaluation of 909 patients from an italian referral center," Inflammatory Bowel Diseases, vol. 16, no. 2, pp. 186-187, 2010. 
[21] K. Matsumura, H. Nakase, S. Yamamoto et al., "Modulation of the Th1/Th2 balance by infliximab improves hyperthyroidism associated with a flareup of ulcerative colitis," Inflammatory Bowel Diseases, vol. 15, no. 7, pp. 967-968, 2009.

[22] T. Inokuchi, Y. Moriwaki, S. Takahashi, Z. Tsutsumi, T. Ka, and T. Yamamoto, "Autoimmune thyroid disease (Graves' disease and Hashimoto's thyroiditis) in two patients with Crohn's disease: case reports and literature review," Internal Medicine, vol. 44, no. 4, pp. 303-306, 2005.

[23] N. Pooran, P. Singh, and S. Bank, "Crohn's disease and risk of fracture: does thyroid disease play a role?" World Journal of Gastroenterology, vol. 9, no. 3, pp. 615-618, 2003.

[24] M. Yakut, Y. Üstün, G. Kabacan, and I. Soykan, "Thyroid disorders in patients with inflammatory bowel diseases," International Journal of Clinical Medicine, vol. 2, no. 2, pp. 89-92, 2011.

[25] H. Oshitani, Y. Wakabayashi, Y. Sawaguchi, H. Koike, and Y. Yoshino, "A case of dermatomyositis followed by multiple mononeuritis, with the history of ulcerative colitis and Basedow's disease," Journal of the Kyorin Medical Society, vol. 12, pp. 47-53, 1981.

[26] M. Chiba, T. Naganuma, M. Gotoh et al., "A case of ulcerative colitis complicated by antibiotic-induced pseudomembranous colitis with a reference to association of Basedow's disease," Gastroenterological Endoscopy, vol. 27, no. 12, pp. 2797-2805, 1985.

[27] H. L. A. Janssen, A. H. M. Smelt, and B. van Hoek, "Graves' hyperthyroidism in a patient with primary sclerosing cholangitis. Coincidence or combined pathogenesis?" European Journal of Gastroenterology \& Hepatology, vol. 10, no. 3, pp. 269-271, 1998.

[28] Y. Terashima, H. Nishiwaki, and T. Suzuki, "A case of ulcerative colitis complicated with refractory Basedow's disease," Hormone to Rinsho, vol. 49, pp. 78-81, 2001.

[29] A. Kohyama, Y. Funayama, K. Fukushima et al., "An operative case of ulcerative colitis associated with hyperthyroidism," Journal of Japanese Society of Gastroenterology, vol. 106, no. 6, pp. 820-825, 2009.

[30] E. Ku, W. Ananthapanyasut, and V. M. Campese, "IgA nephropathy in a patient with ulcerative colitis, Graves' disease and positive myeloperoxidase ANCA," Clinical Nephrology, vol. 77, no. 2, pp. 146-150, 2012.

[31] R. Gómez, A. K. Shetty, A. Vargas et al., "Chronic inflammatory demyelinating polyradiculoneuropathy and Graves' disease in an adolescent with Crohn's disease," Journal of Pediatric Gastroenterology and Nutrition, vol. 29, no. 1, pp. 91-94, 1999.

[32] K. Kanda, Y. Okada, M. Nakai, E. Morita, and Y. Tanaka, "A case of Graves' disease occurred as recrudescence of Crohn's disease," Naika, vol. 94, pp. 397-399, 2004.

[33] S. A. Shah, M. A. Peppercorn, and J. A. Pallotta, "Autoimmune (Hashimoto's) thyroiditis associated with Crohn's disease," Journal of Clinical Gastroenterology, vol. 26, no. 2, pp. 117-120, 1998.

[34] J. A. Carneros, C. Ciriza, Ó. Urquiza, and S. Dajil, "Crohn's disease in a patient with Hashimoto's thyroiditis and Sjögren's syndrome," Medicina Clinica, vol. 119, no. 2, pp. 78-89, 2002.

[35] H. Noto, M. Iimura, T. Karibe et al., "A case of Turner syndrome complicated with Crohn's disease, erythema nodosum and Hashimoto disease," Japanese Journal of Gastroenterology, vol. 103, no. 9, pp. 1039-1043, 2006.

[36] I. Bank and J. O. Busari, "Crohn's disease, autoimmune thyroiditis, and beta-thalassemia trait in an adolescent: an unusual combination of diseases," European Journal of Pediatrics, vol. 167, no. 11, pp. 1343-1346, 2008.

[37] S. Honjo, M. Shiraishi, A. Doyama et al., "A case of small intestine Crohn's disease diagnosed by double balloon endoscopy with Hashimoto's disease," Rinsho Shonikagaku, vol. 60, pp. 3942, 2012.

[38] U. Halac and D. Herzog, "Bardet-Biedl syndrome, Crohn disease, primary sclerosing cholangitis, and autoantibody positive thyroiditis: a case report and a review of a cohort of BBS patients," Case Reports in Medicine, vol. 2012, Article ID 209827, 5 pages, 2012.

[39] J. K. Triantafillidis, S. Durakis, and E. Merikas, "Crohn's disease of the small bowel, complicated by primary biliary cirrhosis, Hashimoto thyroiditis, and Raynaud's phenomenon: favorable response of all disorders to adalimumab treatment," Gastroenterology and Hepatology from Bed to Bench, vol. 6, no. 2, pp. 101105, 2013.

[40] A. J. Greenstein, D. B. Sachar, A. K. Panday et al., "Amyloidosis and inflammatory bowel disease. A 50-year experience with 25 patients," Medicine, vol. 71, no. 5, pp. 261-270, 1992.

[41] Y. Kaku, Y. Kikuchi, Y. Uchida et al., "A case of amyloid goiter with transient hypothyroidism and accelerated uptake of iodine secondary to small-intestine Crohn's disease," Folia Endocrinologica Japonica, vol. 71, p. 854, 1995.

[42] S. Habu, H. Watanobe, K.-I. Kimura, and T. Suda, "A case of amyloid goiter secondary to Crohn's disease," Endocrine Journal, vol. 46, no. 1, pp. 179-182, 1999.

[43] Y. Sudo, N. Kako, K. Ito et al., "A case of amyloid goiter secondary to Crohn's disease treated by total thyroidectomy," Endocrine Surgery, vol. 17, pp. 185-189, 2000.

[44] S. Yamaga, K. Hirakushi, N. Mitsuhata et al., "A case of giant amyloid goiter complicated with tracheal stenosis in a patient with Crohn's disease," Folia Endocrinologica Japonica, vol. 83, p. 342, 2007.

[45] T. Matsui and H. Inoue, "A case of amyloid goiter secondary to Crohn's disease," Igaku Kensa, vol. 57, p. 577, 2008.

[46] G. L. Gallardo, M. P. Moya, M. D. D. Rey, M. J. C. Arroyo, F. M. Dávila, and M. A. Sánchez-Covisa, "Amyloid goiter secondary to crohn's disease," Endocrinología y Nutrición, vol. 56, no. 7, pp. 384-386, 2009.

[47] E. Kazdaghli Lagha, I. M’Sakni, F. Bougrine, B. Laabidi, D. Ben Ghachem, and A. Bouziani, "Amyloid goiter: first manifestation of systemic amyloidosis," European Annals of Otorhinolaryngology, Head and Neck Diseases, vol. 127, no. 3, pp. 108-110, 2010.

[48] B. Febrero, A. Ríos, J. M. Rodríguez, B. Ferri, L. Polo, and P. Parrilla, "Giant amyloid goiter in Crohn's disease," Endocrine Pathology, vol. 23, no. 4, pp. 260-263, 2012.

[49] I. A. Ozemir, C. Bilgic, B. Bayraktar et al., "Amyloid goiter related with Crohn's disease: a rare association: amyloid goiter secondary to Crohn's disease," International Journal of Surgery Case Reports, vol. 5, no. 8, pp. 480-483, 2014.

[50] J. Kawashima, H. Naoe, Y. Sasaki, and E. Araki, "A rare case showing subacute thyroiditis-like symptoms with amyloid goiter after anti-tumor necrosis factor therapy," Endocrinology, Diabetes \& Metabolism Case Reports, vol. 2015, Article ID 140117, 2015.

[51] B. Tunc, L. Filik, A. Ulker, A. Demirbag, and B. Sahin, "Subclinical thyroid disorders and inflammatory bowel disease," Romanian Journal of Gastroenterology, vol. 14, no. 1, pp. 98-99, 2005. 
[52] F. Menconi, C. Marcocci, and M. Marinò, "Diagnosis and classification of Graves' disease," Autoimmunity Reviews, vol. 13, no. 4-5, pp. 398-402, 2014.

[53] L. Bartalena, "Diagnosis and management of Graves disease: a global overview," Nature Reviews Endocrinology, vol. 9, no. 12, pp. 724-734, 2013.

[54] F. Tada, M. Abe, H. Nunoi et al., "Ulcerative colitis complicated with primary biliary cirrhosis," Internal Medicine, vol. 50, no. 20, pp. 2323-2327, 2011.

[55] M. Nakayama, H. Tsuji, J. Shimono et al., "Primary biliary cirrhosis associated with ulcerative colitis," Fukuoka Acta Medica, vol. 92, no. 10, pp. 354-359, 2001.

[56] A. Kettaneh, S. Prevot, A. Biaggi et al., "Hyperthyroidism in two patients with Crohn disease and Takayasu arteritis," Scandinavian Journal of Gastroenterology, vol. 38, no. 8, pp. 901903, 2003.

[57] D. El Fassi, C. H. Nielsen, P. Junker, H. C. Hasselbalch, and L. Hegedüs, "Systemic adverse events following rituximab therapy in patients with Graves' disease," Journal of Endocrinological Investigation, vol. 34, no. 7, pp. el63-e167, 2011.

[58] D. El Fassi, C. H. Nielsen, J. Kjeldsen, O. Clemmensen, and L. Hegedüs, "Ulcerative colitis following B lymphocyte depletion with rituximab in a patient with Graves' disease," Gut, vol. 57, no. 5, pp. 714-715, 2008.

[59] K. Conrad, D. Roggenbuck, and M. W. Laass, "Diagnosis and classification of ulcerative colitis," Autoimmunity Reviews, vol. 13, no. 4-5, pp. 463-466, 2014.

[60] M. Marinò, F. Latrofa, F. Menconi, L. Chiovato, and P. Vitti, "Role of genetic and non-genetic factors in the etiology of Graves' disease," Journal of Endocrinological Investigation, vol. 38, no. 3, pp. 283-294, 2015.

[61] J. Zheng, S. Ibrahim, F. Petersen, and X. Yu, "Meta-analysis reveals an association of PTPN22 C1858T with autoimmune diseases, which depends on the localization of the affected tissue," Genes and Immunity, vol. 13, no. 8, pp. 641-652, 2012.

[62] L.-M. Diaz-Gallo, L. Espino-Paisán, K. Fransen et al., "Differential association of two PTPN22 coding variants with Crohn's disease and ulcerative colitis," Inflammatory Bowel Diseases, vol. 17, no. 11, pp. 2287-2294, 2011.

[63] S. Bank, P. Skytt Andersen, J. Burisch et al., "Polymorphisms in the inflammatory pathway genes TLR2, TLR4, TLR9, LY96, NFKBIA, NFKB1, TNFA, TNFRSF1A, IL6R, IL10, IL23R, PTPN22, and PPARG are associated with susceptibility of inflammatory bowel disease in a Danish cohort," PLoS ONE, vol. 9, no. 6, Article ID e98815, 2014.

[64] M. Zhang, J. Ni, W.-D. Xu et al., "Association of CTLA-4 variants with susceptibility to inflammatory bowel disease: a meta-analysis," Human Immunology, vol. 75, no. 3, pp. 227-233, 2014.

[65] S. Zeissig, B. Petersen, M. Tomczak et al., "Early-onset Crohn's disease and autoimmunity associated with a variant in CTLA4," Gut, vol. 64, no. 12, pp. 1889-1897, 2015.

[66] F. Blanco-Kelly, F. Matesanz, A. Alcina et al., "CD40: novel association with Crohn's disease and replication in multiple sclerosis susceptibility," PLOS ONE, vol. 5, no. 7, Article ID el1520, 2010.

[67] P. Caturegli, A. De Remigis, and N. R. Rose, "Hashimoto thyroiditis: clinical and diagnostic criteria," Autoimmunity Reviews, vol. 13, no. 4-5, pp. 391-397, 2014.

[68] M. T. Bardella, L. Elli, S. D. Matteis, I. Floriani, V. Torri, and L. Piodi, "Autoimmune disorders in patients affected by celiac sprue and inflammatory bowel disease," Annals of Medicine, vol. 41, no. 2, pp. 139-143, 2009.

[69] C. N. Bernstein, A. Wajda, and J. F. Blanchard, “The clustering of other chronic inflammatory diseases in inflammatory bowel disease: a population-based study," Gastroenterology, vol. 129, no. 3, pp. 827-836, 2005.

[70] M. Najafi, M. M. Zamani, N. Rezaei, and M. Sabbaghian, "Autoimmunity in inflammatory bowel disease: a case of ulcerative colitis with diabetes mellitus, autoimmune hepatitis and autoimmune hypothyroidism," Turkish Journal of Pediatrics, vol. 54, no. 6, pp. 651-653, 2012.

[71] O. A. Paoluzi, P. Crispino, M. Rivera et al., "Skeletal muscle disorders associated with inflammatory bowel diseases: occurrence of myositis in a patient with ulcerative colitis and Hashimoto's thyroiditis-case report and review of the literature," International Journal of Colorectal Disease, vol. 21, no. 5, pp. 473-477, 2006.

[72] F. Topal, E. Senel, S. Akbulut, F. Topal, and Y. Dölek, "A new combination of multiple autoimmune syndrome? Coexistence of vitiligo, autoimmune thyroid disease and ulcerative colitis," Dermatology Reports, vol. 3, article e19, 2011.

[73] S. Obata, K. Higashi, F. Kawano et al., "A case of Turner's syndrome with idiopathic thrombocytopenic purpura, and Hashimoto's thyroiditis detected in the course of ulcerative colitis," Nihon Shokakibyo Gakkai Zasshi, vol. 91, no. 4, pp. 899903, 1994.

[74] H. Nishimura, M. Kino, S. Kubo, and K. Kawamura, "Hashimoto's thyroiditis and ulcerative colitis in a patient with Turner's syndrome," The Journal of the American Medical Association, vol. 254, no. 3, pp. 357-358, 1985.

[75] M. J. Goldacre and O. O. Seminog, "Turner syndrome and autoimmune diseases: record-linkage study," Archives of Disease in Childhood, vol. 99, no. 1, pp. 71-73, 2014.

[76] A. A. Alfadda, R. M. Sallam, G. E. Elawad, H. Aldhukair, and M. M. Alyahya, "Subacute thyroiditis: clinical presentation and long term outcome," International Journal of Endocrinology, vol. 2014, Article ID 794943, 7 pages, 2014.

[77] H. Ikenoue, K. Okamura, T. Kuroda, K. Sato, M. Yoshinari, and M. Fujishima, "Thyroid amyloidosis with recurrent subacute thyroiditis-like syndrome," Journal of Clinical Endocrinology and Metabolism, vol. 67, no. 1, pp. 41-45, 1998.

[78] Y. Horai, T. Miyamura, K. Shimada et al., "A case of Takayasu's arteritis associated with human leukocyte antigen A24 and B52 following resolution of ulcerative colitis and subacute thyroiditis," Internal Medicine, vol. 50, no. 2, pp. 151-154, 2011.

[79] M. Kaklamanos, D. Thomas, D. Pikazis, and G. Kaltsas, "Thyroid-specific changes following treatment with biological therapies in patients with rheumatic diseases," Endocrine, vol. 50, pp. 146-153, 2015.

[80] Y. Sethi, A. Gulati, I. Singh, S. Rao, and N. Singh, "Amyloid goiter: a case of primary thyroid amyloid disease," Laryngoscope, vol. 121, no. 5, pp. 961-964, 2011.

[81] A. D’Antonio, R. Franco, L. Sparano, G. Terzi, and G. Pettinato, "Amyloid goiter: the first evidence in secondary amyloidosis. Report of five cases and review of literature," Advances in Clinical Pathology, vol. 4, no. 2, pp. 99-106, 2000.

[82] H. Kimura, S. Yamashita, K. Ashizawa, N. Yokoyama, and S. Nagataki, "Thyroid dysfunction in patients with amyloid goitre," Clinical Endocrinology, vol. 46, no. 6, pp. 769-774, 1997.

[83] T. Ohta, M. Nishi, Y. Furukawa et al., "A case of painful amyloid goiter secondary complicated with ulcerative colitis," Folia Endocrinologica Japonica, vol. 84, p. 445, 2008. 
[84] A. C. Moss, A. M. Brennan, A. S. Cheifetz, and M. A. Peppercorn, "Thyroid cancer and Crohn's disease: association or coincidence?” Inflammatory Bowel Diseases, vol. 12, no. 1, pp. 79-80, 2006.

[85] N. Evgenikos and J. G. Stephen, "Papillary thyroid carcinoma associated with ulcerative colitis," Postgraduate Medical Journal, vol. 72, no. 852, pp. 621-622, 1996.

[86] G. Casella, E. Antonelli, C. Di Bella et al., "Papillary thyroid cancer and ulcerative colitis," Gastroenterology and Hepatology from Bed to Bench, vol. 6, no. 1, pp. 52-54, 2013.

[87] B. S. Wong, D. W. Larson, T. C. Smyrk, and A. S. Oxentenko, "Perforated Meckel's diverticulum presenting with combined bowel and urinary obstruction and mimicking Crohn's disease: a case report," Journal of Medical Case Reports, vol. 4, article 264, 2010.

[88] G. G. Ginsberg, Z. D. Goodman, and J. H. Lewis, "A 22-year-old man with thyroid cancer and cholestatic liver disease," Seminars in Liver Disease, vol. 11, no. 1, pp. 64-71, 1991.

[89] J. M. Byun, S. K. Baek, H.-J. Yoon et al., "Thyroid cancer and T lymphoblastic leukemia in crohn disease: a case report and literature review," Laboratory Medicine, vol. 46, no. 2, pp. 140$145,2015$.

[90] J. K. Triantafillidis, "Ulcerative colitis associated with extranodal marginal zone B-cell thyroid lymphoma of mucosaassociated lymphoid tissue and hashimoto thyroiditis: description of a case," Annals of Gastroenterology, vol. 26, article 92, 2013.

[91] R. García Arroyo, R. Carrión Galindo, J. A. Arranz Arija, C. González San Segundo, L. Martín Fragueiro, and E. Flores Sanudo, "Primary large cell thyroid lymphoma. Report of 6 cases treated with chemotherapy," Medicina Clinica, vol. 103, no. 4, pp. 136-139, 1994.

[92] W. J. Mack, S. Preston-Martin, L. Bernstein, and D. Qian, "Lifestyle and other risk factors for thyroid cancer in Los Angeles County females," Annals of Epidemiology, vol. 12, no. 6, pp. 395-401, 2002.

[93] Y. Yano, T. Matsui, F. Hirai et al., "Cancer risk in Japanese Crohn's disease patients: investigation of the standardized incidence ratio," Journal of Gastroenterology and Hepatology, vol. 28, no. 8, pp. 1300-1305, 2013.

[94] I. S. Sonu, W. Blonski, M. V. Lin, J. Lewis, F. Aberra, and G. R. Lichtenstein, "Papillary thyroid cancer and inflammatory bowel disease: is there a relationship?" World Journal of Gastroenterology, vol. 19, no. 7, pp. 1079-1084, 2013.

[95] K. Khamassi, H. Jaafoura, F. Masmoudi, R. Lahiani, L. Bougacha, and M. Ben Salah, "Ectopic lingual thyroid," Case Reports in Pediatrics, vol. 2015, Article ID 252357, 4 pages, 2015. 


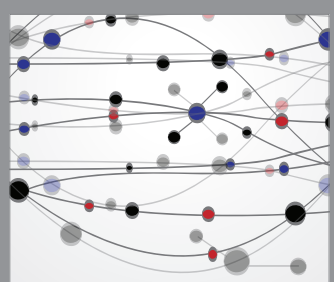

The Scientific World Journal
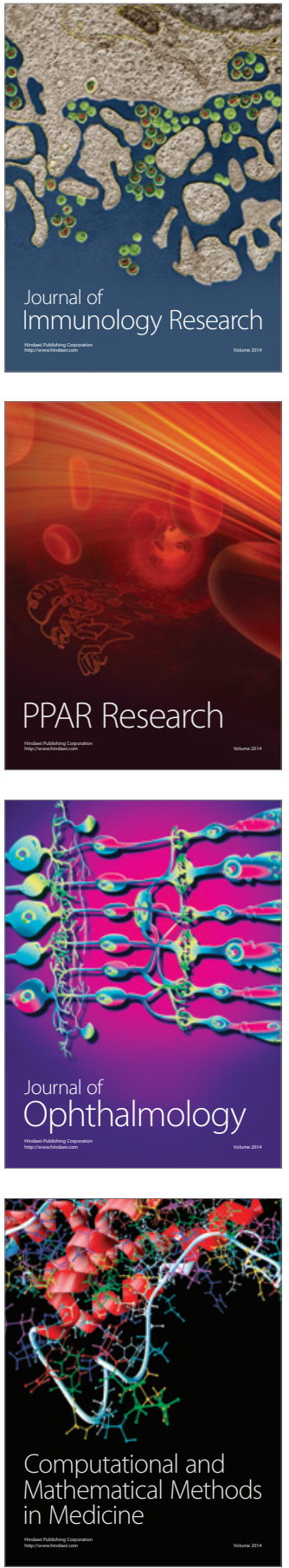

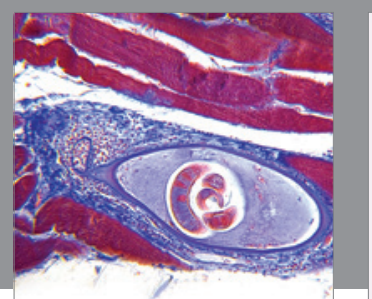

Gastroenterology Research and Practice

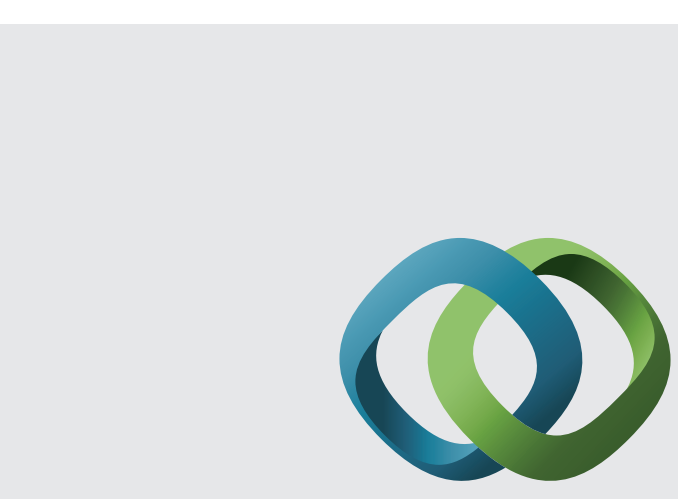

\section{Hindawi}

Submit your manuscripts at

http://www.hindawi.com
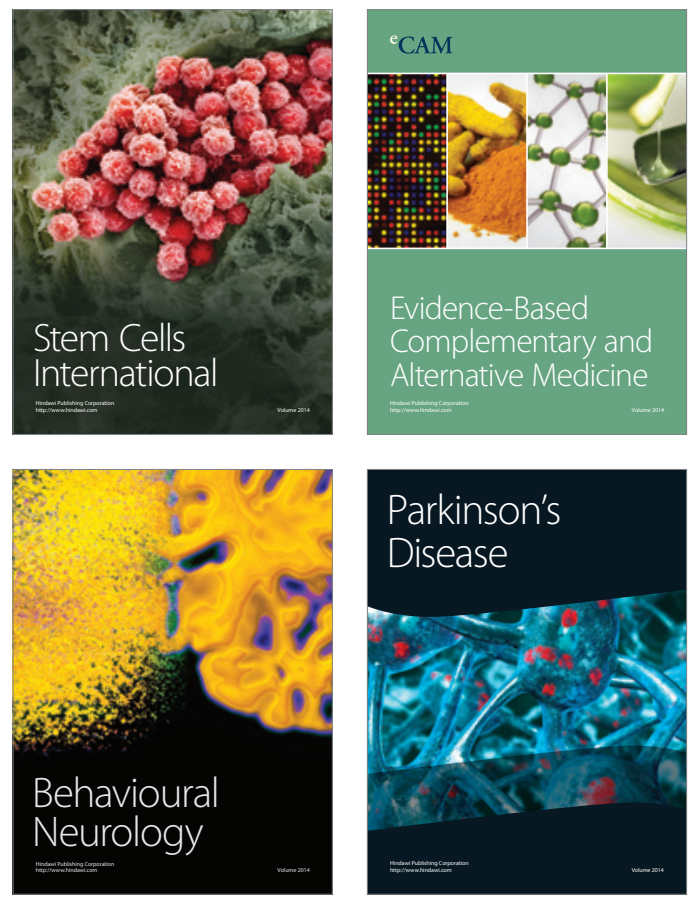
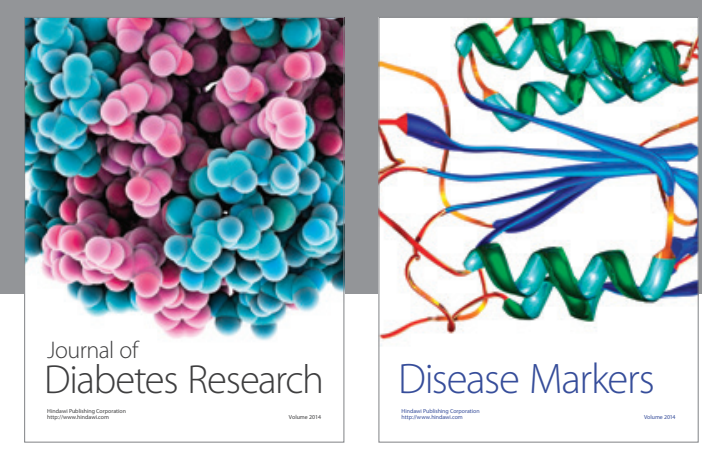

Disease Markers
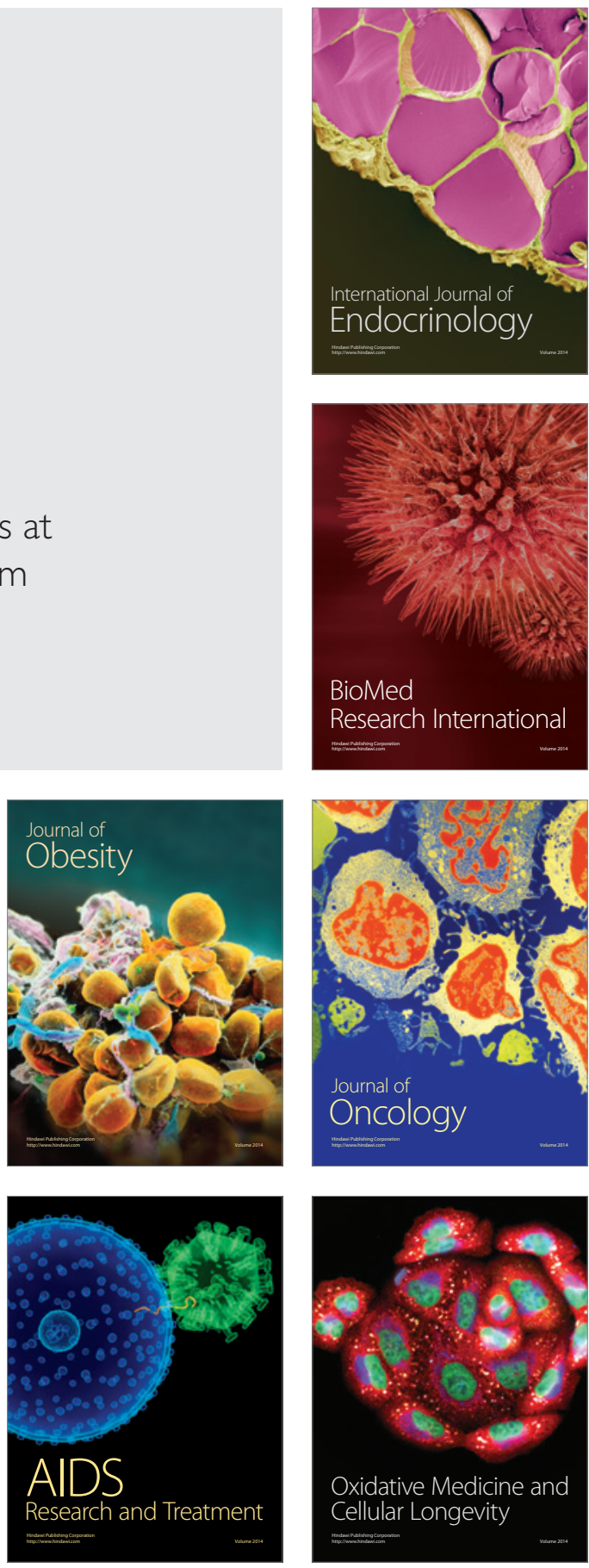